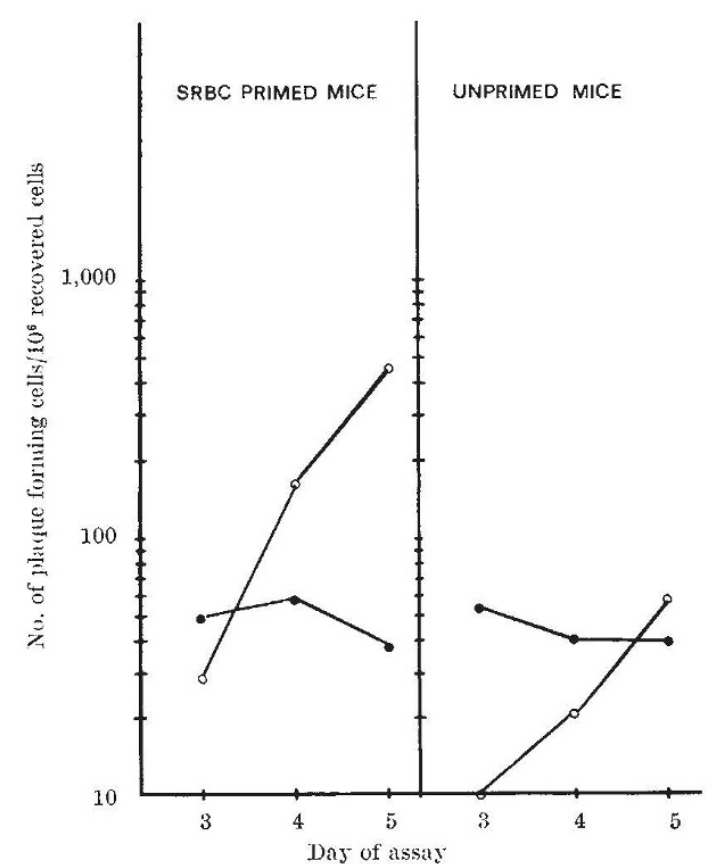

Fig. 2. Effect of prior immunization by carrier red cell; plaque formers are assayed on NIP-DRBC. Immunized Fl hybrid $\mathrm{C} 57 \mathrm{Bl} / 6 \times \mathrm{DBA} 2$ miee were primed by an injection of $\mathrm{SRBC} 44$ days before setting up cultures. Antigens used in culture: $O$, XIP-SRBC; none.

there was no diminution in the plaque forming cells, which suggested that at least two cells were involved in the anti-hapten response, and that the carrier specific cell in the spleen from a primed animal was in excess over the hapten specific cell.

Cells synthesizing antibodies to a hapten can be induced consistently in vitro by hapten coupled to red cells and by using spleen cells from mice immunized previously with the red cell carrier. When spleen cells from unimmunized mice are used, the response is variable. Because prior immunization with the carrier erythrocyte seems to be essential for hapten response some correlation between the in vitro response to the hapten and to the carrier cell might be expected. This was found insofar as the highest responders to one specificity were the highest to the other and the lowest also the lowest, but intermediato values did not correspond. This may be related to the fact that dilution of the primed cells with cells from unimmunized animals showed that the primed cells were in excess. The specificity of induction by the hapten seems clear, as does the specificity of the carrier cell.

The results with the hapten carricr complex could be interpreted in terms of current hypotheses? as being a consequence of cooperation of cells primed against the carrier with cells capable of synthesizing antibodies to the hapten.

We thank Mrs Joan Rankin for technical assistance, and Drs N. A. Mitchison, P. Plotz, G. Fleet and A. Munro for haptens. E. S. L. received partial research support from the US Public Health Service.

\section{S. TROWBRIDGE}

E. S. LENNOX*

R. R. PORTER

MRC Immunochemistry Unit,

Department of Biochernistry,

South Parks Road,

Oxford.

Received August 7,1970 .

* Permanent address: Salk Institute, San Diego, USA.

${ }^{2}$ Mishell, R. I., and Dutton, R. W., J. Exp. Med., 126, 423 (1967).

${ }^{2}$ Segal, S., Globerson, A., Feldman, M., Haimovich, J., and Sela, M., J. Exp. Med., 181, $93(1970)$
${ }^{3}$ Kettman, J., and Dutton, R. W., J. Immunol., 104, 1558 (1970).

4 Brownstone, A., Mitchison, N. A., and Pitt-Kivers, R., Immunology, 10, 465 (1966).

Pasanen, V. J., and Mäkelä, O., Immunology, 16, 399 (1969).

${ }^{6}$ Kennedy, J. C., Treadwell, P. E., and Lennox, E. S., J. Exp. Med. (in the press).

Mitchison, N. A., Rajewsky, K., and Taylor, R. B., in Developmental Aspects of Antibody Formation and Structure (edit. by Sterzl, J., and Riha, O. I.) (Academic Press, New York, in the press).

\section{Mechanism for Joining $\boldsymbol{V}$ and $\boldsymbol{C}$ Genes}

I would like to suggest an alternative to the mechanism suggested by Gally and Edelman ${ }^{1}$ for the joining of the $V$ gene to the $C$ gene. This mechanism has three principal features: (1) no "cross-over" event (for excision and integration) is required; (2) the joined product, the coupled $V C$ into one gene, could either be an RNA or a DNA molecule; and (3) the $C$ gene in its joining end must have a few redundant bases which are almost identical to the joining end of the $V$ gene.

Consider the genes $V$ and $C$ in Fig. 7 of Gally and Edelman's article and assume that the attachment site is the overlap region in the joining ends of $V$ and $C$ genes (Fig. la). Let the transcription enzyme transcribe opposite strands of $V$ and $C$ genes to generate a partially double stranded RNA molecule. Alternatively, these could be as in Fig. 1b, single stranded pieces of DNA. At this point a repair enzyme together with a ligase converts it into a double stranded molecule which would now replicate with the help of a replicase (Fig, 1c). A mechanism to generate diversity could be introduced by errors in the repair process; in this case the heterozygous molecule must segregate very early.

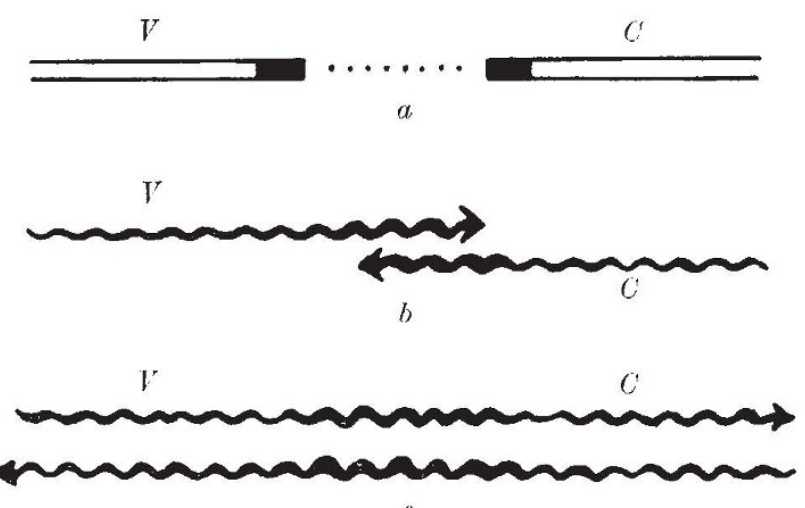

Fig. 1. - The joining of $V$ and $C$ genes. See text for explanation.

This self replicating, intermediate template might be a special inheritable material of somatic cells producing immunoglobulin in very large amounts. If such templates are present they would perhaps be unique to immunoglobulin producing cells or cells similar to it-which require to produce many adventitious specificities built into molecules which also share common specificities.

Another mechanism of joining $V$ and $C$ genes is suggested in Fig. 5 of ref. 1 ; excision of the genetic region between the right end of any $V$ gene and the loft end of the $C$ gene will achieve the joining by only one "crossover" event.

SIDDHARTHA SARKAR

The Salk Institute,

San Diego,

California 92112 .

Received August 13, 1970.

1 Gally, J. A., and Edelman, G. M., Nature, 227, 341 (1970). 